\title{
Berliner Stiftung für Dermatologie (BSD) Wissenschaftliches Jahres-Symposium am 12. Mai 2007 in Berlin
}

\author{
$8^{\text {th }}$ Annual Scientific Symposium of the Berlin Foundation for Dermatology, Berlin, May $12^{\text {th }}, 2007$
}

Autor

Institution

\section{C. Geilen}

Berliner Stiftung für Dermatologie, Dresdner Bank AG, Pariser Platz 6, 10877 Berlin
Bibliografie

Dol $10.1055 / \mathrm{s}-2007-967004$

Akt Dermatol 2007; 33:

407-409 ๑ Georg Thieme

Verlag KG Stuttgart · New York ISSN 0340-2541

Korrespondenzadresse

Prof. Dr. med. Dr. rer. nat. Christoph C. Geilen

Dermatologie am Kurfürstendamm Kurfürstendamm 45 10719 Berlin prof.geilen@dermalink.de
Im Mai dieses Jahres fand das diesjährige Symposium der Berliner Stiftung für Dermatologie (BSD) in den schönen Räumen der Dresdner Bank am Pariser Platz in Berlin statt, wo die offizielle Vergabe der Jahrespreise der Stiftung an die Preisträger 2007 erfolgte und Stipendien sowie Forschungsförderungsmittel für Nachwuchswissenschaftler aus dem In- und Ausland vergeben wurden.

Es ist inzwischen die achte Veranstaltung dieser Art, die die BSD mit Erfolg absolviert. Die Berliner Stiftung für Dermatologie wurde 1999 von den Herren Professor Dr. Günter Stüttgen und Professor Dr. Prof. Dr. h.c. Constantin E. Orfanos gegründet mit dem Ziel, die wissenschaftliche Forschung auf dem Gebiet der Dermatologie und ihrer Grenzgebiete mittels Preisen, Stipendien und Forschungszuschüssen zu fördern, wobei der Schwerpunkt in der Weiterbildung und den Forschungsaktivitäten jüngerer Kollegen liegt. Insbesondere das Gebiet der Erarbeitung therapeutisch relevanter Forschungserkenntnisse und ihre Bedeutung für die klinische Praxis stehen im Mittelpunkt der Zielsetzung. Ein anderes wichtiges Förderungsziel der Stiftung ist die Dermato-Venerologie auf internationaler Ebene, einschließlich des Austausches von Wissenschaftlern zwecks Lehre und Fortbildung.

Die Berliner Stiftung blickt nur wenige Jahre nach ihrer Gründung bereits auf eine beachtliche Zahl von Preisträgern und Stipendiaten zurück, die die Aktivität der Stiftung unterstreichen. Insgesamt 34 junge Kollegen und Wissenschaftler aus 19 Nationen gehören bisher zu den Geförderten († Abb. 1).

Immerhin 21 Stipendien ermöglichten jungen Kollegen, sich in Deutschland über eine befristete Zeit (meist 6 Monate bis 1 Jahr) in dermatologischen Exzellenzzentren wissenschaftlich-klinisch weiterzubilden, in den dazugehörigen Forschungslaboratorien spezielle Techniken zu erlernen sowie Tagungen und Kongresse im deut- schen Sprachraum zu besuchen. Drei ausländische Stipendiaten nutzten die finanzielle Unterstützung der Stiftung, um in Kooperation mit einer Institution ihrer Heimat an einer deutschen Universität mit einem Forschungsthema zu promovieren.

In den vergangenen Jahren lag ein Schwerpunkt der Stiftungsarbeit in der Förderung der Dermatologie in den osteuropäischen Ländern. In den Jahren nach der politischen Öffnung im Osten Europas war dort der Bedarf an Forschungsförderung und Unterstützung aller Art besonders groß. Viele der bisherigen BSD-Stipendiaten kamen dementsprechend aus Bulgarien, Rumänien, Ungarn, Tschechien, Litauen und Estland. In seiner letzten Sitzung im Rahmen des diesjährigen Symposiums hat das Kuratorium der Berliner Stiftung beschlossen, einen neuen Schwerpunkt auf die Entwicklung der Dermatologie und die dermato-venerologische Weiterbildung in Afrika zu setzen. Dieses gesonderte „Afrika-Programm“ der Stiftung soll zur Verbesserung der Versorgung von Patienten mit Hautkrankheiten und sexuell übertragbaren Infektionen in den afrikanischen Ländern beitragen, die heute unter den anderen Fächern verteilt zum Teil brach liegt oder von fachfremden Kollegen in ungenügender Weise wahrgenommen wird. Insbesondere die Vorbeugung und Behandlung der sexuell übertragbaren Infektionen, einschließlich der grassierenden HIV-Infektion, steht in den Ländern der Subsahara im Vordergrund und ist eine große Herausforderung für die dortigen Sozialsysteme. Hierzu soll vonseiten der Berliner Stiftung unter anderem ein Afrika-Stipendium für 2008/09 als Förderungsinstrument eingesetzt werden, mit dessen Hilfe qualifizierte junge Ärzte aus Europa mit dermatologischen Kenntnissen bzw. Erfahrung, bevorzugt Dermatologen, ihr Wissen in Ländern der Subsahara-Region weitergeben und die dortige dermato-venerologische Versorgung unterstützen sollen. Eine entsprechende Aus- 


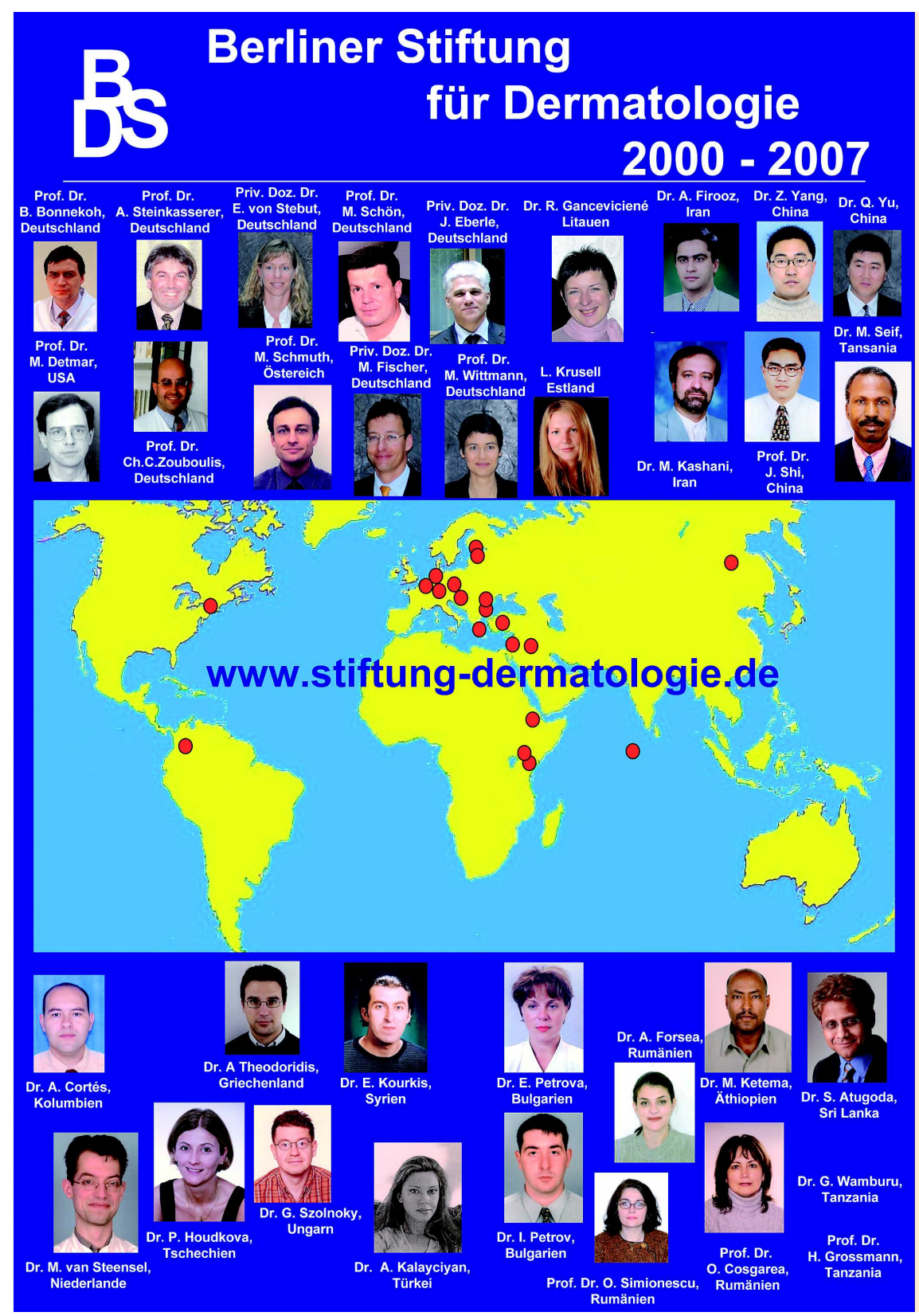

Abb. 1 Berliner Stiftung für Dermatologie 2000-2007.

34 Preisträger und Stipendiaten aus 19 Ländern wurden bisher durch die Berliner Stiftung für Dermatologie für ihre Leistungen auf dem Gebiet der Dermatologie geehrt und in ihrer wissenschaftlichen Arbeit unterstützt. schreibung für dieses Stipendium wird gesondert erfolgen, doch können sich eventuelle Interessenten bereits jetzt an die Geschäftsführung der Stiftung wenden: karin.kohler@dresdnerbank.com oder constantin.orfanos@charite.de.

Auf dem diesjährigen Symposium der Stiftung in Berlin wurden zwei junge Dermatologinnen, Frau Professor Dr. Miriam Wittmann (Hannover) und Frau Privatdozentin Dr. Ruth Esther von Stebut (Mainz), geehrt und aufgrund ihrer Forschungsleistungen mit dem Preis der Berliner Stiftung für Dermatologie 2007 ausgezeichnet ( Abb. 2). Frau Professor Wittmann stellte dem Auditorium ihr Forschungsgebiet in einem hochinteressanten Vortrag mit dem Titel „Der Beitrag von Keratinozyten an der Pathogenese des kutanen Lupus erythematodes “vor, während Frau Privatdozentin von Stebut im Vortrag „Immunologie der Leishmaniose: Stand und Perspektiven“ ihr wissenschaftliches Arbeitsgebiet eindrucksvoll und umfassend darstellte.

Zusätzlich zur Preisvergabe wurde in diesem Jahr ein Forschungsprojekt von Herrn Privatdozent Dr. Matthias Fischer (Halle) gefördert, der den Stand der Forschung und seine bisherigen
Ergebnisse in einem Vortrag mit dem Thema „Transmitterfunktionen in der menschlichen Epidermis am Beispiel keratinozytärer Glutamatrezeptoren“ umriss. Darüber hinaus erhielt Herr Dr. Qiang Yu aus China ein BSD-Foschungsstipendium, mit dessen Hilfe er sich über 6 Monate (ggf. 1 Jahr) in Deutschland aufhalten kann, um sich klinisch und wissenschaftlich auf dem Gebiet der Akneforschung in der Hautklinik Dessau bei Herrn Prof. Dr. Christos Zouboulis zu betätigen. Herr Dr. Mebratu Ketema (Äthiopien), der derzeit im Regional Dermatology Training Centre in Moshi, Tansania, in der dermatologischen Weiterbildung tätig ist, erhielt die Mittel, um sich über 1 Monat in der UniversitätsHautklinik Leipzig bei Herrn Prof. Dr. Jan Simon aufzuhalten und fortzubilden, sowie eine Sachmittelunterstützung, um seine Diplomarbeit auf dem Gebiet der HIV-Infektion fortzuführen.

Die Preisträgerinnen und Stipendiaten, die Mitglieder des Kuratoriums der Stiftung sowie zahlreiche Teilnehmer hatten im Anschluss an die Vorträge und bei einem kleinen Buffet Gelegenheit zum Gedankenaustausch und zu ausführlichen Diskussionen mit den Preisträgern und Stipendiaten. Die BSD hat sich zur 


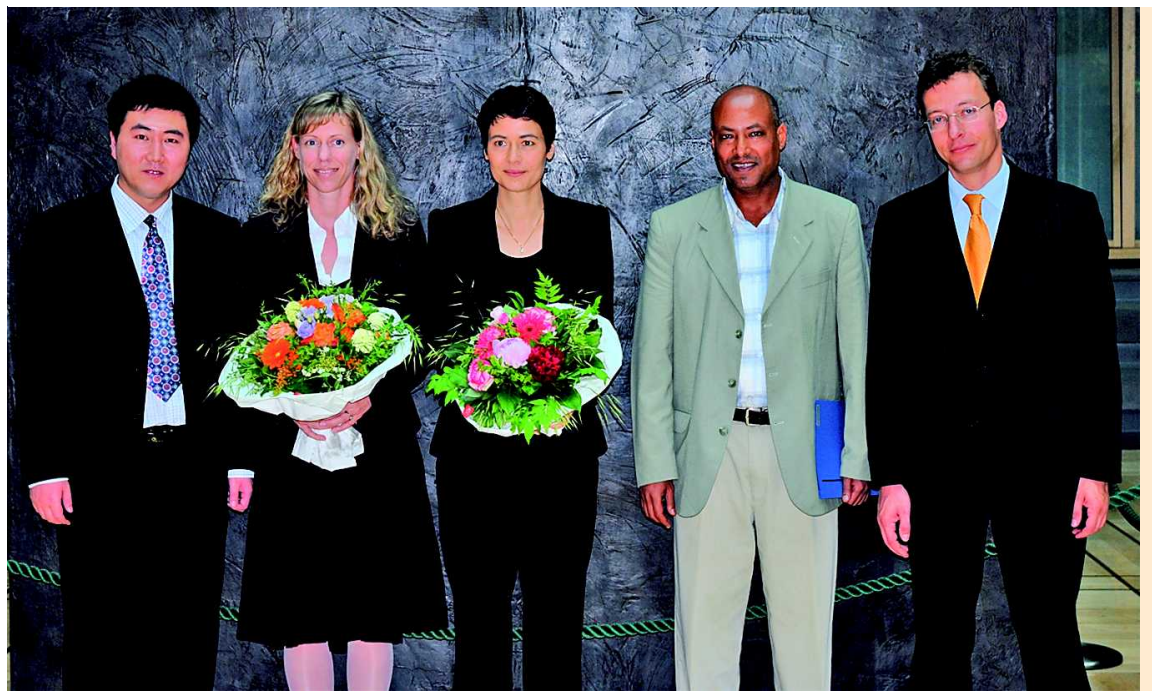

Abb. 2 Preisträger und Stipendiaten der Berliner Stiftung für Dermatologie (BSD) 2007. Von links nach rechts: Dr. Qiang Yu (Xushou, China), PD Dr. Ruth Esther von Stebut (Mainz, Deutschland), Prof. Dr. Miriam Wittmann (Hannover, Deutschland), Dr. Mebratu Ketema (Äthiopien; jetzt Moshi, Tansania), PD Dr. Matthias Fischer (Halle, Deutschland).

Regel gemacht, auch den persönlichen Kontakt zu ihren Alumni zu pflegen und sie zu den kommenden Veranstaltungen einzuladen bzw. sie in speziellen Fragestellungen als Berater heranzuziehen.

Neben dem ehemaligen Präsidenten der Deutschen Dermatologischen Gesellschaft (DDG), Professor Dr. Harald Gollnick (Magdeburg), Herrn Professor Dr. Sergji Goerdt (Mannheim) und Herrn Professor Dr. Jan Simon (Leipzig), die ohnehin dem Kuratorium der Stiftung angehören, konnte die BSD auch den neuen Generalsekretär der DDG, Herrn Professor Dr. Rudolf Stadler (Minden) sowie weitere dermatologische Ordinarien, wie Professor Dr. Alexander Kapp (Hannover), Professor Dr. Jürgen Knop (Mainz) und Professor Dr. Ingrid Moll (Hamburg), begrüßen. Dass neben dem wissenschaftlichen Anspruch auch die Bedeutung der klinischen Aspekte bei den Symposien der Berliner Stiftung nicht vernachlässigt wird, zeigte die rege Teilnahme von Klinikassistenten und niedergelassenen Kolleginnen und Kollegen. Die Teilnahme an den Symposien der Stiftung ist stets frei und allen, die dieses Jahr dabei waren, sei für ihr Interesse gedankt.

Nachdrücklich ist der Dresdner Bank AG zu danken, die ihre eindrucksvollen Räumlichkeiten am Pariser Platz im Zentrum Berlins, mit bester Aussicht auf das Brandenburger Tor, erneut und großzügig der Stiftung zur Verfügung gestellt hat. Man fühlte sich im Herzen Deutschlands, sowohl die in- wie auch die ausländischen Gäste haben den Blick genossen. Die Berliner Stiftung für Dermatologie hofft, dass das nächste 9. BSD-Symposium, das im Frühjahr 2008 wiederum in Berlin stattfinden wird, eine ähnlich große Resonanz haben wird.

Zum Schluss soll nicht vergessen werden zu erwähnen, dass die Förderungsmöglichkeiten einer Stiftung wie der BSD maßgeblich von ihrer finanziellen Ausstattung abhängig sind. Insofern soll der vorliegende Beitrag auch eine Anregung für eventuelle Spenden und Zustiftungen sein, die ein wichtiger Garant für zukünftige, erfolgreiche Aktivitäten der BSD im Interesse unseres Faches, gerade in den Entwicklungsländern, werden können. Jeder Beitrag wird dabei helfen, das gesetzte Ziel, die Förderung der Dermato-Venerologie, zu erreichen. Insbesondere für unser Afrika-Programm werden Mittel dankbar angenommen.

Weitere Informationen zur Berliner Stiftung für Dermatologie und den aktuellen Terminen sind unter www.stiftung-dermatologie.de zu finden. 